\title{
Targeted pharmacotherapy after somatic cancer mutation
}

\section{screening [version 1; peer review: 1 approved]}

\author{
Thomas M. Polasek (D1), Karen Ambler2, Hamish S. Scott², Michael J. Sorich1, \\ Peter A. Kaub², Andrew Rowland1', Michael D. Wiese³, Ganessan Kichenadasse ${ }^{4}$ \\ ${ }^{1}$ Department of Clinical Pharmacology, Flinders Medical Centre and Flinders University, Adelaide, Australia \\ ${ }^{2}$ Department of Genetics and Molecular Pathology, SA Pathology, Adelaide, Australia \\ ${ }^{3}$ School of Pharmacy and Medical Science, University of South Australia, Adelaide, Australia \\ ${ }^{4}$ Department of Medical Oncology, Flinders Centre for Innovation in Cancer, Adelaide, Australia
}

V1 First published: 30 Jun 2016, 5:1551

https://doi.org/10.12688/f1000research.9040.1

Latest published: 20 Sep 2016, 5:1551

https://doi.org/10.12688/f1000research.9040.2

\section{Open Peer Review}

Approval Status

1

2

version 2

(revision)

20 Sep 2016

version 1

30 Jun 2016

1. Andrew A. Somogyi, University of Adelaide,

Adelaide, Australia

2. Nimit Singhal, Royal Adelaide Hospital,

Adelaide, Australia

Any reports and responses or comments on the article can be found at the end of the article.

Corresponding author: Thomas M. Polasek (tom.polasek@flinders.edu.au)

Competing interests: No competing interests were disclosed.

Grant information: The author(s) declared that no grants were involved in supporting this work.

Copyright: @ 2016 Polasek TM et al. This is an open access article distributed under the terms of the Creative Commons Attribution License, which permits unrestricted use, distribution, and reproduction in any medium, provided the original work is properly cited. Data associated with the article are available under the terms of the Creative Commons Zero "No rights reserved" data waiver (CC0 1.0 Public domain dedication).

How to cite this article: Polasek TM, Ambler K, Scott HS et al. Targeted pharmacotherapy after somatic cancer mutation screening [version 1; peer review: 1 approved] F1000Research 2016, 5:1551 https://doi.org/10.12688/f1000research.9040.1

First published: 30 Jun 2016, 5:1551 https://doi.org/10.12688/f1000research.9040.1 


\section{Introduction}

Over the last 20 years the molecular profiles of many solid tumours have been characterised. The discovery of specific variants in critical proteins that influence cancer pathogenesis has seen the development of 'targeted pharmacotherapy' - drugs that selectively inhibit unique molecular targets in tumour cells. Compared to traditional cytotoxic agents, targeted drugs have considerable benefits in the treatment of cancer, including improved response rates and less toxicity ${ }^{1}$.

This field of cancer therapeutics is rapidly evolving with several hundred ongoing clinical trials. However, there are no local guidelines in Australia to inform the prescribing of targeted pharmacotherapy. As a consequence, clinicians often use resources from pharmaceutical companies, conference presentations, journal publications or recommendations from other countries, such as the US National Comprehensive Cancer Network (NCCN) guidelines, for their clinical practice. Although the NCCN guidelines are not always directly applicable for practice in Australia, these are reviewed annually, are freely available (www.nccn.org), and have best practice recommendations for targeted pharmacotherapy use in selected cancers.

In addition to the well documented role of estrogen/progesterone receptor and HER-2 testing in selecting therapies for breast cancer, three other important cancers in Australia, malignant melanoma, non-small cell lung cancer (NSCLC) and metastatic colorectal cancer (mCRC), now have targeted drugs available for treatment based on genetic testing. Dabrafenib, with or without trametinib, is used for malignant melanoma with activating $B R A F$ mutations ('BRAF positive') $)^{2}$. Patients with NSCLC that harbours activating EGFR mutations ('EGFR positive') are recommended the EGFR inhibitors erlotinib or gefitinib ${ }^{3}$. Two monoclonal antibodies that also inhibit EGFR (cetuximab and panitumumab) significantly improve survival in patients with mCRC that is $R A S$ wild-type (WT), whereas those with mutations in $R A S$ are essentially insensitive ${ }^{4}$. Bevacizumab is a selective inhibitor of VEGR that is also used in $\mathrm{mCRC}$ but response rates are independent of $R A S$ status i.e., genetic testing is often not necessary for treatment decisions. Bevacizumab is frequently used first-line in combination with chemotherapy regimens such as FOLFOX, FOLFIRI and CapeOX ${ }^{5}$. Figure 1 shows the 2015 NCCN recommendations for targeted pharmacotherapy based on the molecular profiles of the cancers investigated in this study ${ }^{6-9}$.

OncoFOCUS $^{\mathrm{TM}}+$ KIT is a somatic cancer mutation screen offered by SA Pathology (www.sapathology.sa.gov.au) for clinicians in South Australia. The test analyses the oncogenes KRAS, NRAS, EGFR, $B R A F$ and KIT. Clinically significant mutations in these genes are reported as either 'no mutation detected' (WT) or as a specific

\section{A. Malignant Melanoma}

BRAF positive

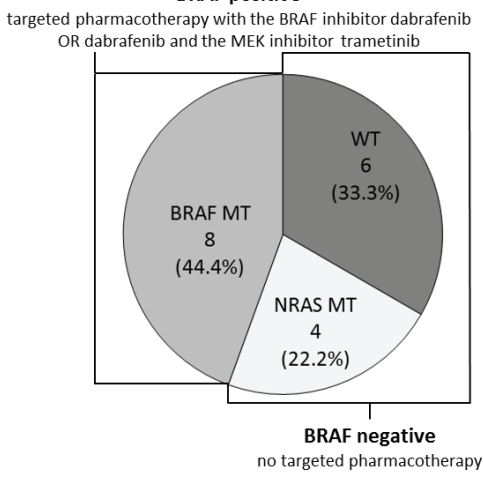

\section{B. Non-Small Cell Lung Cancer}
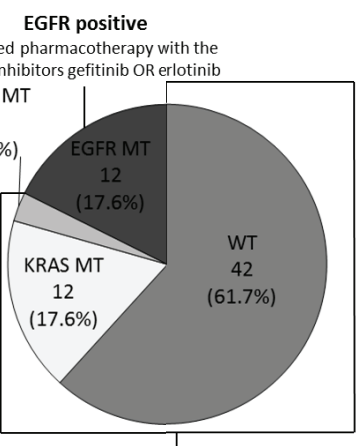

EGFR negative no targeted pharmacotherapy

\section{Metastatic Colorectal Cancer}

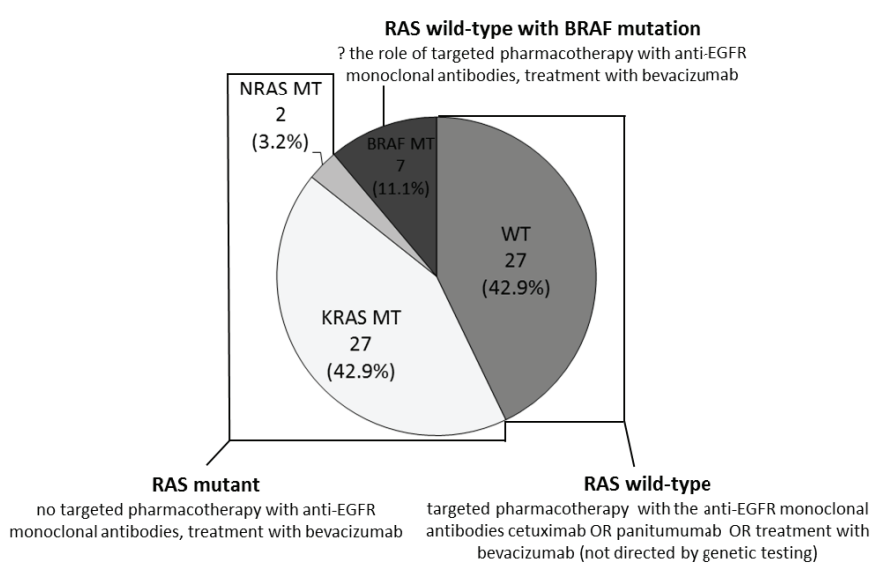

Figure 1. OncoFOCUS ${ }^{\mathrm{TM}}+K I T$ results, molecular cancer classifications, and the $2015 \mathrm{NCCN}$ guideline recommendations for targeted pharmacotherapy. 
mutation e.g., BRAF V600E. Screening with OncoFOCUS ${ }^{\mathrm{TM}}+K I T$ has recently been introduced at the Flinders Centre for Innovation in Cancer (FCIC), an academic healthcare centre located in the southern suburbs of Adelaide that specialises in research and treatment of cancer. Given this introduction into clinical practice, and the lack of local prescribing guidelines, the aim of this study was to audit targeted pharmacotherapy use after screening against the latest NCCN recommendations.

\section{Methods}

A retrospective chart-based audit of OncoFOCUS ${ }^{\mathrm{TM}_{+}}+$KIT results and targeted pharmacotherapy use was conducted. Ethics approval for the study was granted by the Southern Adelaide Human Research Ethics Committee (application 137.15). Inclusion criteria were: $\geq 18$ years, diagnosis of malignant melanoma, advanced NSCLC or mCRC, record of attendance at the FCIC in 2014, and OncoFOCUS ${ }^{\mathrm{TM}_{+}}$KIT results reported in 2014. The electronic patient system OACIS was searched for genetic test results and relevant discharge summaries, multi-disciplinary team meeting summaries and electronic and/or hardcopy case notes were reviewed to determine pharmacotherapy use. In a small number of cases (21), information about medications used in private practice was confirmed with the treating oncologist. Retrieval of data was conducted over a 3 month period between June-August 2015. Results were presented as descriptive data or as a percentage.

\section{Results}

Sixty percent (90/149) of the cohort were male and 40\% (59/149) were female, with a mean average age of 67.6 years (range 34 to 91 years). At the audit cut-off date, $48.3 \%$ (72) were alive, $49.7 \%$ (74) were deceased and the living status of $2.0 \%$ (3) could not be determined. There were similar numbers of patients with NSCLC (68) and mCRC (63) but a smaller number of patients with malignant melanoma (18).

OncoFOCUS $^{\mathrm{TM}}+$ KIT results for patients with malignant melanoma, NSCLC and mCRC are shown in Figures 1A-C, respectively. All patients were KIT WT. Importantly, the cohort had similar cancer mutation rates as previously reported. Forty four percent with malignant melanoma had an activating $B R A F$ mutation (40-60\% reported ${ }^{10}$ ), $17.6 \%$ had EGFR-positive NSCLC (10-20\% reported $\left.^{11}\right)$, and $46 \%$ had $R A S$ mutant mCRC (40\% reported $\left.{ }^{12}\right)$. These data suggest that the FCIC cohort is representative of the wider population.

Of the 149 patients included, only 6 patients $(3.8 \%)$ were excluded from the analysis of targeted pharmacotherapy use due to incomplete records. Figure 2 shows the percentage of patients who received or did not receive a targeted drug according to NCCN guidelines. Appropriately, almost all patients not indicated for targeted pharmacotherapy did not receive targeted pharmacotherapy (99\%, 100/101). Of the 42 patients in the total cohort indicated for targeted therapy, $79 \%$ (33/42) received such treatment according to NCCN guidelines (Figure 2). Of the 25 patients with mCRC that was RAS WT, 36\% (9/25) had targeted pharmacotherapy directed by OncoFOCUS ${ }^{\mathrm{TM}}+$ KIT with an anti-EGFR drug (8 cetuximab, 1 panitumumab), 52\% (13/25) received bevacizumab, and $12 \%(3 / 25)$ did not receive a targeted drug in contrast to $\mathrm{NCCN}$

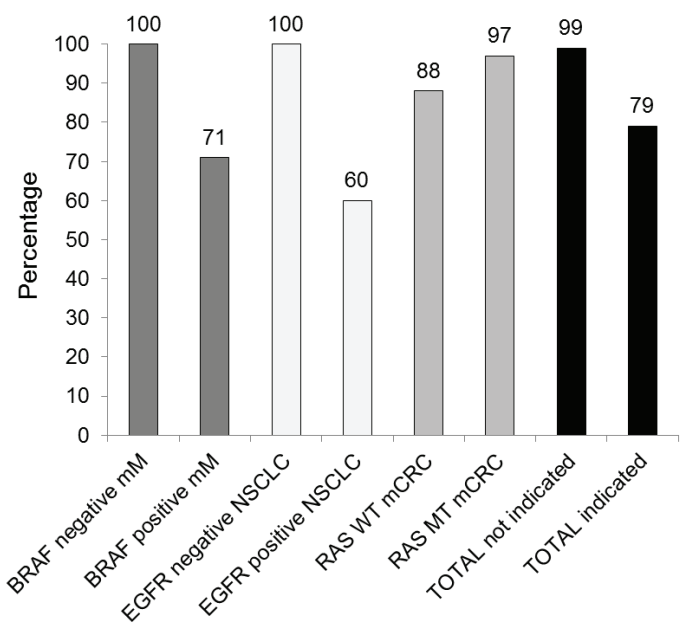

Figure 2. Percentage of patients who received or did not receive targeted pharmacotherapy according to NCCN guidelines after OncoFOCUS ${ }^{\mathrm{TM}}+K I T$ screening.

guidelines. If bevacizumab in RAS WT mCRC is excluded, $48 \%$ (20/42) of the total indicated cohort received appropriate targeted drugs following OncoFOCUS ${ }^{\mathrm{TM}}+$ KIT screening i.e., required genetic test results for a targeted drug to be prescribed.

Dataset 1. OncoFOCUS screening raw data

http://dx.doi.org/10.5256/f1000research.9040.d127508

Frequency of oncogene mutations and targeted pharmacotherapy in malignant melanoma, advanced non-small cell lung cancer, and metastatic colorectal cancer.

\section{Discussion}

This study is the first to report utilisation rates of targeted pharmacotherapy after OncoFOCUS ${ }^{\mathrm{TM}_{+}}+$KIT screening. As expected, patients who were not indicated for targeted pharmacotherapy did not receive such treatment. In contrast, the use of targeted drugs directed by OncoFOCUS ${ }^{\mathrm{TM}_{+}}+$KIT screening was relatively low $(48 \%)$.

This result may be explained by factors that are independent of $\mathrm{OncoFOCUS}^{\mathrm{TM}}+$ KIT results. First, the use of bevacizumab in $\mathrm{mCRC}$ does not require genetic testing - it is considered equivalent to cetuximab and panitmumumab in RAS WT mCRC and was given first-line to most patients with mCRC at FCIC $^{6}$. This is confusing because bevacizumab is a targeted drug by definition, selectively inhibiting VEGR. Second, targeted drugs for NSCLC and $\mathrm{mCRC}$ were subsidised by the Australian Pharmaceutical Benefits Scheme (PBS) in 2014 as second-line only. Thus, patients on first-line chemotherapy appropriately did not receive targeted drugs, despite having mutations suggesting they may benefit from such treatment. During 2014, anti-EGFR drugs became indicated for first-line treatment of EGFR-positive NSCLC and were funded by the $\mathrm{PBS}^{13}$. Likewise, cetuximab and panitumumab are now PBS-subsidised as first-line treatment in $R A S \mathrm{WT} \mathrm{mCRC}^{13}$. Not 
differentiating between first- and second-line targeted pharmacotherapy is a major limitation of the study (note that half the cohort was still alive at the audit cut-off date, precluding a more complete analysis of the temporal relationships between screening and targeted pharmacotherapy use). Third, a number of patients had genetic testing close to the end of life. These patients were considered too unwell for further oncology treatment, or declined targeted drugs when offered, preferring to transfer to palliative care.

The exact role of targeted drugs for some of the cancer mutations reported by OncoFOCUS ${ }^{\mathrm{TM}_{+}}+K I T$ is unclear. For example, approximately $5-9 \%$ of colorectal cancers (7.9\% in this study) are characterised by a specific mutation in the BRAF gene (V600E) which causes constitutive activity, in theory bypassing inhibition by cetuximab and panitumumab and potentially making them insensitive $^{14}$. In the colon cancer NCCN guidelines, BRAF mutation testing is currently optional and not part of decision making for anti-EGFR drugs ${ }^{6}$. A recent meta-analysis suggests that there is currently insufficient evidence to conclude that patients with mCRC harbouring BRAF mutations should be denied anti-EGFR therapy over concerns of poor efficacy ${ }^{15}$. However, there are conflicting views on whether BRAF status should influence use of anti-EGFR therapy ${ }^{16,17}$, and hence some clinicians may potentially utilise BRAF status to make treatment decisions. This highlights the difficulty of auditing medical oncology prescribing where guidelines and the underlying evidence are rapidly evolving.

The OncoFOCUS ${ }^{\mathrm{TM}}+K I T$ screening panel is currently limited to five oncogenes. The status of other oncogenes that may influence treatment decisions is determined separately. For example, patients with NSCLC are also tested for $A L K$ rearrangements, and if positive are eligible for treatment with crizotinib (although it is not currently PBS-subsidised for this indication $)^{8}$. Once the importance of emerging genetic alternations is established in these cancers, such as MET amplifications, ROS1 and RET rearrangements, and
HER 2 mutations, the OncoFOCUS ${ }^{\mathrm{TM}_{+}}+K I T$ screening panel could be expanded to facilitate more complete molecular diagnosis.

In conclusion, this study showed that most patients at the FCIC receive pharmacotherapy for their cancer according to NCCN guidelines (93\%), and that the results of a somatic cancer mutation screening test are applied reasonably well to drug selection. Precision medicine approaches are of increasing importance when directing pharmacotherapy in medical oncology.

\section{Data availability}

F1000Research: Dataset 1. OncoFOCUS screening raw data, 10.5256/f1000research.9040.d127508 ${ }^{18}$

Author contributions

Participated in research design: Polasek and Kichenadasse

Collected data: Polasek and Ambler

Performed data analysis: Polasek

Wrote or contributed to writing of the manuscript: Polasek, Scott, Sorich, Kaub, Rowland, Wiese and Kichenadasse

\section{Competing interests}

No competing interests were disclosed.

\section{Grant information}

The author(s) declared that no supporting grants were involved in this work.

\section{Acknowledgements}

We thank Glenice Cheetham, Musei Ho and Madelyn Zawitkowski at SA Pathology for assay development and conducting the genetic analyses.
1. Simon R, Rowchowdhury S: Implementing personalized cancer genomics in clinical trials. Nat Rev Drug Discov. 2013; 12(5): 358-369. PubMed Abstract | Publisher Full Text

2. Atkinson V: Medical management of malignant melanoma. Aust Prescr. 2015; 38(3): 74-78.

PubMed Abstract | Publisher Full Text | Free Full Text

3. Giaconne G: Epidermal growth factor receptor inhibitors in the treatment of non-small-cell lung cancer. J Clin Oncol. 2005; 23(14): 3235-3242. PubMed Abstract | Publisher Full Text

4. Sorich MJ, Wiese MD, Rowland A, et al.: Extended RAS mutations and antiEGFR monoclonal antibody survival benefit in metastatic colorectal cancer: a meta-analysis of randomized, controlled trials. Ann Oncol. 2015; 26(1): 13-21. PubMed Abstract | Publisher Full Text

5. Lv C, Wu S, Zheng D, et al:: The efficacy of additional bevacizumab to cytotoxic chemotherapy regimens for the treatment of colorectal cancer: an updated meta-analysis for randomized trials. Cancer Biother Radiopharm. 2013; 28(7): 501-509.

PubMed Abstract | Publisher Full Text | Free Full Text

6. NCCN. NCCN Clinical Practice Guidelines in Oncology. Colon Cancer Version 3. 2015. 2015.

7. NCCN. NCCN Clinical Practice Guidelines in Oncology. Melanoma Version 3. 2015. 2015.
8. NCCN. NCCN Clinical Practice Guidelines in Oncology. Non-Small Cell Lung Cancer Version 7. 2015. 2015.

9. NCCN. NCCN Clinical Practice Guidelines in Oncology. Rectal Cancer Version 3. 2015. 2015

10. Menzies AM, Haydu LE, Visintin L, et al.: Distinguishing clinicopathologic features of patients with V600E and V600K BRAF-mutant metastatic melanoma Clin Cancer Res. 2012; 18(12): 3242-3249. PubMed Abstract | Publisher Full Text

11. Hirsch FR, Bunn PA Jr: EGFR testing in lung cancer is ready for prime time. Lancet Oncol. 2009; 10(5): 432-433. PubMed Abstract | Publisher Full Text

12. Roth AD, Tejpar S, Delorenzi M, et al.: Prognostic role of KRAS and BRAF in stage II and III resected colon cancer: results of the translational study on the PETACC-3, EORTC 40993, SAKK 60-00 trial. J Clin Oncol. 2010; 28(3): 466-474. PubMed Abstract | Publisher Full Text

13. Health Do: Pharmaceutical Benefits Scheme. 2015. Accessed 31/7/2015. Reference Source

14. Tol J, Nagtegaal ID, Punt CJ: BRAF mutation in metastatic colorectal cancer N Engl J Med. 2009; 361(1): 98-99. PubMed Abstract | Publisher Full Text

15. Rowland A, Dias MM, Wiese MD, et al:: Meta-analysis of BRAF mutation as a 
predictive biomarker of benefit from anti-EGFR monoclonal antibody therapy for RAS wild-type metastatic colorectal cancer. Br J Cancer. 2015; 112(12):

1888-1894.

PubMed Abstract | Publisher Full Text | Free Full Text

16. Cremolini C, Di Maio M, Petrelli F, et al.: BRAF-mutated metastatic colorectal cancer between past and future. Br J Cancer. 2015; 113(11):

1634-1635.

PubMed Abstract | Publisher Full Text | Free Full Text
17. Rowland A, Dias MM, Wiese MD, et al.: Reply: Comment on 'Meta-analysis of BRAF mutation as a predictive biomarker of benefit from anti-EGFR monoclonal-antibody therapy for RAS wild-type metastatic colorectal cancer'. Br J Cancer. 2015; 113(11): 1635.

PubMed Abstract | Publisher Full Text | Free Full Text

18. Polasek T, Ambler K, Scott H, et al:: Dataset 1 in: Targeted pharmacotherapy after somatic cancer mutation screening. F1000Research. 2016 Data Source 


\section{Open Peer Review}

\section{Current Peer Review Status:}

\section{Version 1}

Reviewer Report 24 August 2016

https://doi.org/10.5256/f1000research.9728.r15849

(c) 2016 Somogyi A. This is an open access peer review report distributed under the terms of the Creative Commons Attribution License, which permits unrestricted use, distribution, and reproduction in any medium, provided the original work is properly cited.

\section{Andrew A. Somogyi}

Discipline of Pharmacology, School of Medicine, Faculty of Health Sciences, University of Adelaide, Adelaide, SA, Australia

The title suitably addresses the content of the article; in the Abstract, after the second last line, a comment should be made regarding the $52 \%$ who were screened by the test but did not receive the targeted drugs. The content of the article in general is of a high standard, it could be improved to the casual and inexpert reader by clarifying where KIT, KRAS and NRAS fit within the overall picture as BRAF deals with MM, EGFR with NSCLC and RAS with MCRC; and of the 6 exclusions the number in each cohort (targeted versus no targeted) could be stated. Finally, Figure 2 is unclear and a disconnect between the figure (BRAF, EGFR, RAS positive, negative) and the legend (...received or did not receive targeted pharmacotherapy), to include actual numbers and not just percentages would also make it clearer. The conclusions are most appropriate and it succinctly highlights the complex interplay between testing, prescribing, guidelines, evidence and funding all of which are rapidly changing but are not connected, all of which have universal implications.

Competing Interests: No competing interests were disclosed.

\section{I confirm that I have read this submission and believe that I have an appropriate level of expertise to confirm that it is of an acceptable scientific standard.}

Author Response 12 Sep 2016

Thomas Polasek, Flinders Medical Centre and Flinders University, Adelaide, Australia

We thank Prof Somogyi for these very useful comments and suggestions regarding our manuscript. We have now uploaded version 2 to incorporate changes based on the review as described below:

1. 'after the second last line, a comment should be made regarding the $52 \%$ who were screened by the test but did not receive the targeted drugs'. We have now added the following in the abstract, 'with the remaining 52\% (22/42) prescribed drugs 
independent of the screening results for various reasons.'

2. 'by clarifying where KIT, KRAS and NRAS fit within the overall picture'. In the introduction we have now added that KIT testing is used for selecting imatinib for metastatic melanoma e.g. 'whereas imatinib can be used for KIT-mutated melanoma'. We have not added further comments about the differences between KRAS and NRAS, since they are considered together in targeted drug selection for mCRC (as already described in the manuscript by referring to RAS), and mutations in these for metastatic melanoma and NSCLC are not currently indications for targeted drug selection.

3. 'and of the 6 exclusions the number in each cohort (targeted versus no targeted) could be stated'. We have already stated that these 6 could not be included in the analysis due to incomplete records (Results first line paragraph 3).

4. 'Figure 2 is unclear and a disconnect between the figure (BRAF, EGFR, RAS positive, negative) and the legend (...received or did not receive targeted pharmacotherapy), to include actual numbers and not just percentages would also make it clearer'. We have added a new Figure 2 to make the distinction clearer, including raw data.

Competing Interests: No competing interests to declare.

The benefits of publishing with F1000Research:

- Your article is published within days, with no editorial bias

- You can publish traditional articles, null/negative results, case reports, data notes and more

- The peer review process is transparent and collaborative

- Your article is indexed in PubMed after passing peer review

- Dedicated customer support at every stage

For pre-submission enquiries, contact research@f1000.com 